\title{
EVALUATION OF SENTINEL-2/MSI IMAGERY PRODUCTS LEVEL-2A OBTAINED BY THREE DIFFERENT ATMOSPHERIC CORRECTIONS FOR MONITORING SUSPENDED SEDIMENTS CONCENTRATION IN MADEIRA RIVER, BRAZIL.
}

\author{
Santos, D. R. A e ${ }^{1 *}$, Martinez, J. M. ${ }^{12 *}$, Tristan Harmel ${ }^{2 *}$, Borges, H.D ${ }^{1 *}$, Roig $\mathrm{H}^{1}$. \\ ${ }^{1}$ Instituto de Geociências, Universidade de Brasília - IG-UnB, Brasília-DF, Brasil \\ ${ }^{2}$ Géosciences Environnement Toulouse (GET), UMR5563, Institut de Recherche pour le Développement (IRD)/Centre National de \\ la Recherche Scientifique (CNRS)/Université Toulouse 3, 14 Avenue Edouard Belin, 31400 Toulouse, France
}

KEY WORDS: Reflectance, atmospheric correction, suspended sediment concentration, GRS, MAJA, Sen2Cor, HYBAM, Amazon

\begin{abstract}
:
Data provided by spatial sensors combined with remote sensing techniques and analysis of the optical properties of waters allow the mapping of the suspended sediment concentration (SSC) in aquatic bodies. For this, estimation models require data with the lowest possible amount of atmospheric artifacts. In this study we compared the water remote sensing reflectance (Rrs) of the Santo Antônio Hydroelectric Power Plant reservoir in Porto Velho-RO, Brazil, after applying three different atmospheric corrections algorithms in Sentinel-2/MSI imagery products. The atmospheric corrected reflectances of the MODIS sensor were also used for reference. SSC was calculated with models based on the red and near-infrared (NIR) bands over three distinct regions of the reservoir. Reflectance data showed significant variations for Sentinel-2, bands 4 and 8a, and MODIS, bands RED and IR, when different atmospheric correction algorithms were used. SSC maps and estimates were produced to show sediment load variation as a function of hydrological regime. The analyzes showed that the SSC estimates done with Sentinel-2 / MSI satellite images using GRS (Glint Remove Sentinel) atmospheric correction presented an average difference of $27.3 \%$ and were the closest to the in situ measurements. SSC estimates from MODIS products were around 34.6\% different from estimates made using the GRS atmospheric correction applied to Sentinel-2 / MSI products.
\end{abstract}

\section{INTRODUCTION}

Remote sensing data has been widely used to study the optical properties of waters and to support analyzes of the water bodies sedimentary behavior (Cavali et al. 2019; Palmer et al. 2014). An advantageous mechanism to estimate suspended sediment concentrations (SSC) in water bodies is the application of bio-optical algorithms, which, from data provided by orbital sensors, derive parameters from aquatic constituents (Ogashwara, 2015). Such data must be free of artifacts such as clouds, water vapor, aerosols, shadows and reflections of sunlight and sky. Atmospheric correction models are usually used to convert top of atmosphere reflectance to surface reflectance.

Villar, et. al. (2013) and Martinez et. al. (2015) used in situ water surface spectral reflectance and atmospheric correction data from the MODIS sensor to develop and calibrate a model for monitoring sediment dynamics in the Madeira River and the Amazon basin.

In light of the recent Sentinel-2 mission, launched in 2016, specific atmospheric correction models have been proposed considering the sensor new features and configurations. However, how these different models impact the retrieval of water quality parameters remains to be studied.

The MAJA atmospheric correction (Haggole et. al. 2017) introduces a multitemporal correction to better detect variations from atmospheric processes while SEN2COR (Müller$\mathrm{W}, 2015)$ uses a library of radiation transfer data for different parameters, generating a scene classification to produce the corrected images. Both algorithms were developed for the French and European space agencies respectively, and provide generic atmospheric corrections of Sentinel-2 images for continental surfaces, without particular specification for continental waters.
The GRS algorithm offers exclusive removal of the sunglint effect on the aquatic body (Harmel, et. Al. 2018), valid for continental and coastal waters.

This study used three Sentinel-2 satellite images from the Santo Antônio hydroelectrical power plant (HPP) reservoir region acquired over three distinct hydrological periods - rising waters, flood and dry - to compare the Rrs values after the application of the atmospheric corrections - GRS, MAJA and Sen2Cor. A comparison with the Rrs values of MODIS products was also performed.

Due to the presence of clouds and different spatial resolutions, the comparison of Sentinel-2 / MSI x MODIS data occurred at the nearest dates in which the extraction of pure water pixels was possible in the sampling regions.

Additionally, the SSC estimates retrieved with the different satellite products were compared with water samplings realized in the Madeira River by the international monitoring network SO HYBAM (Geodynamical, hydrological and biogeochemical control of erosion/alteration and material transport in the Amazon, Orinoco and Congo basins) maintained by the Federal University of Amazonas (UFAM) and the IRD (Institut de Recherche pour le Développement) since 2003 in Brazil.

\section{DATA AND METHODS}

\subsection{The study area}

The area considered is located in the downstream part of the Santo Antônio HPP reservoir, located on the Madeira River, in the Brazilian state of Rondônia-RO, encompassing

* diego.alvesesantos@gmail.com; jean-michel.martinez@ird.fr; tristan.harmel@get.omp.eu; henriquedantasborges@gmail.com; roig@unb.br 
different hydro-sedimentary processing, and denominated as L1 as shown in Figure 1.

Three images were selected as they presented limited cloud coverages. After removing cloud pixels, cloud shadows and negative values resulting from postprocessing artefacts, 408,375 pixels were analyzed for $\mathrm{L} 120170628,58,471$ pixels for $\mathrm{L}_{20180119}$ and 191,614 pixels for $\mathrm{L}_{20180424}$ Subsequently, smaller regions were delimited in the L1 region, upstream (U1) and downstream (D1) of the HPP dam and in the flooded backwater areas (igarapés) region (IGP) with darker waters for detailed study of the satellite products.

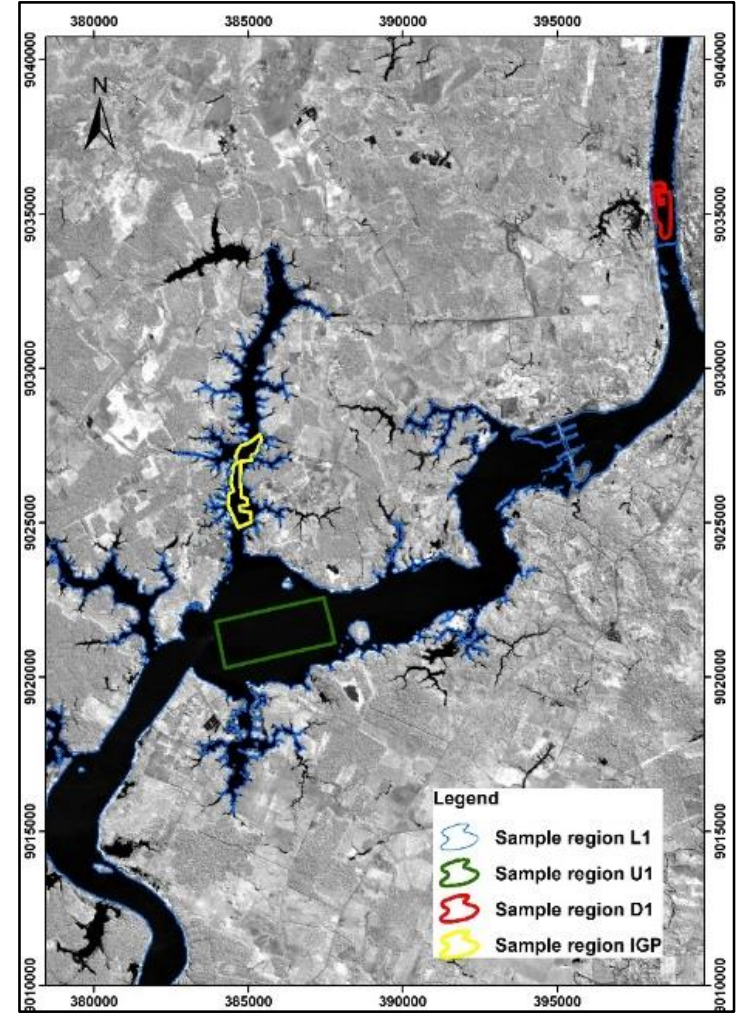

Figure 1 - Location of the regions analysed with Sentinel-2 images

\subsection{Data}

Sentinel-2 Mission MSI data were acquire (https://scihub.copernicus.eu/) and Theia Land Data Center (https://theia.cnes.fr/atdistrib/rocket/\#/home) portals, including images from 06/27/2017 (dry season), 01/19/2018 (rising waters) and 04/24/2018 (flood peak). MODIS MOD09Q1 8-day composite surface reflectance products that provide two radiometric bands at Infrared(IR) (841-876 nm) and at red (620$670 \mathrm{~nm})$ at $250 \mathrm{~m}$ resolution were downloaded for three dates close to the Sentinel-2 acquisitions: 06/26/2017, 01/17/2018 and $04 / 23 / 2018$.

The spectral responses of the Sentinel-2 satellite MSI sensor in bands 4, centered at $664.6 \mathrm{~nm}$, with $10 \mathrm{~m}$ spatial resolution and band $8 \mathrm{a}$, centered at $864.7 \mathrm{~nm}$ and $20 \mathrm{~m}$ spatial resolution, were further analyzed after applying the different atmospheric corrections: GRS, MAJA and Sen2Cor. As the GRS product has a spatial resolution of $20 \mathrm{~m}$, this resolution was chosen as the reference resolution. MAJA products were resampled to $20 \mathrm{~m}$ using the nearest neighbor pixel method, and Sen2cor outputs were set to the same resolution.

SSC sample data collected every 10 days from the HYBAM monitoring station 15400000 at the Madeira River surface were used to assess the accuracy of the satellite-derived SSC estimates.

\subsection{RMSEr calculations}

The reflectances values assessed from the the Sentinel-2 products (Table 1) were compared using the Relative Root Mean Square Error (RMSEr) (Eq. 1), using the average reflectance of the GRS or Sen2Cor products as a reference for each band and for each date.

Where:

$$
\operatorname{RMSEr}=\frac{\frac{\sqrt{\sum_{i=1}^{n}(x-y)^{2}}}{n}}{\bar{x}}
$$
a reference

$$
\mathrm{n}=\text { pixel number, }
$$

$\bar{x}=$ mean Rrs calculated using either GRS or Sen2Cor as

\subsection{SSC Calculations}

To calculate SSC from Rrs spectra, the in situ hyperspectral radiometric database presented by Villar et al. (2013) and completed by Martinez et al. (2015) was used. For the MSI bands, hyperspectral data were convoluted with the spectral response function (SRF) of bands 4 and 8a. Eq. 2 describes the Sentinel-2 data model.

$$
\operatorname{CSS}=945,1 \cdot\left(\frac{b 8 a}{b 4}\right)^{1.9463}
$$

Villar et al. (2013):

For MODIS data, we used Eq. 3 as published by

$$
\text { CSS }=2,94 \cdot\left(\frac{I R}{R E D}\right)^{1,020}
$$

\section{RESULTS AND DISCUSSION}

\subsection{Comparison between Sentinel-2 reflectances estimated} from GRS, MAJA and Sen2cor atmospheric corrections

Comparing the band 4 Sentinel-2 / MSI reflectances (Table 1) resulting from each processing atmospheric corrections, we observe that the MAJA and Sen2Cor products show close behavior and that both processing show distinct values in relation to the GRS processing. RMSEr between the MAJA and Sen2Cor reflectances for the 06/28/2017, $01 / 19 / 2018$ and $04 / 24 / 2018$ images confirms the similarity with $1.75 \%, 5.68 \%$ and $3.12 \%$ respectively. The largest difference between the three processings was found for band 4, in April 2018, between Sen2cor and GRS (23.39\%).

On the $06 / 28 / 2017$, the highest similarity was found among all comparisons, with a RMSEr of $1.75 \%$ between MAJA and Sen2Cor products.

Overall, the highest RMSEr (up to $25 \%$ ) occur when comparing Sen2cor and MAJA processing with products from GRS processing, which is likely caused by the additional removal of the sunglint by GRS.

For the $8 \mathrm{a}$ band, the differences between the Sen2Cor and MAJA to GRS products remain above $10 \%$. 
However, there is a reversal of the behaviour, as the RMSE's between Sen2Cor / GRS (11.26\%) and MAJA / GRS (11.96\%) are closer, while the comparison MAJA / Sen2Cor presents higher RMSEr (21.98\%) in January 2019. For this band, the absorption of light by pure water is strong, leading to lower Rrs values and lower Signal/Noise ratio, which might reduce the differences between the processings.

\begin{tabular}{|c|c|c|c|c|c|c|}
\hline \multirow{2}{*}{ Date } & \multicolumn{5}{|c|}{ RMSEr Reflectances $(\%)$} \\
\cline { 2 - 7 } & \multicolumn{3}{|c|}{ B4 } & \multicolumn{3}{c|}{ B8a } \\
\cline { 2 - 7 } & Sen2Cor X GRS & MAAA X GRS & MAJA X Sen2Cor & Sen2Cor X GRS & MAAA x GRS & MAAA X Sen2Cor \\
\hline 20170628 & 11,46 & 12,03 & 1,75 & 11,26 & 12,08 & 16,69 \\
\hline 20180119 & 21,33 & 16,40 & 5,68 & 13,07 & 14,70 & 21,98 \\
\hline 20180024 & 23,40 & 20,69 & 3,12 & 18,34 & 11,96 & 8,08 \\
\hline
\end{tabular}

Table 1 - RMSEr's L1 region reflectances referenced by mean reflectance GRS and Sen2Cor

Figure 2 compares the reflectance values for band 4 processed using MAJA (x-axis) and Sen2Cor (y-axis) for all the water pixels. The igarapés regions (green dots), formed by small streams with black waters present the lowest reflectance values. The pixels matching the Madeira River main stream (brown pixels), show the highest reflectance values. The best correlations when comparing the reflectances resulting from the application of the three different atmospheric corrections for bands 4 and $8 \mathrm{a}$ on the analyzed dates were for Sen2Cor x MAJA reflectances, similarly to the RMSEr resulting from the comparisons. However, a greater dispersion is observed for the highest reflectances measured by band 4 , above $3.5 \%$ (Figure 3 ). It should be taken into account that in this region the pixel diversity is higher, covering a larger reservoir sampling area. This characteristic appears also for measurements between these corrections when compared to those offered by GRS processing for band 4 .

For band $8 \mathrm{a}$, located in a spectral region of higher absorption than band 4 , there is a reduction in the dispersion from the highest to the lowest reflectances, mainly in June, and a slight variation in the slope of the curves in the GRS $x$ Sen2Cor and GRS $x$ MAJA diagrams on all dates in the 0.008 to 0.018 reflectances values range.

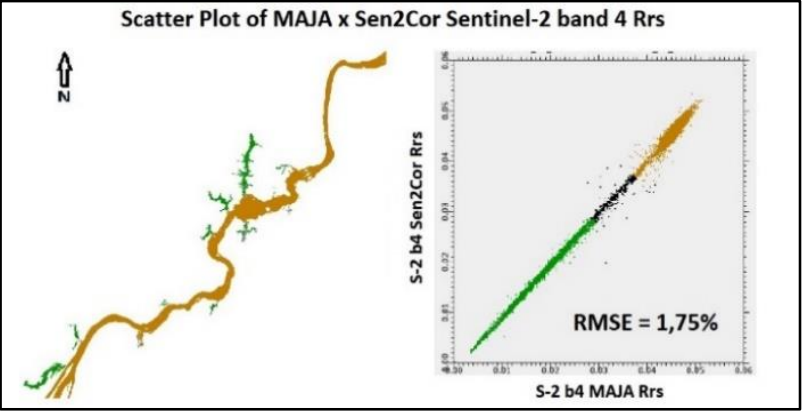

Figure 2. Reflectance by reservoir region

3.2 Comparison between Sentinel-2 mean reflectance estimated by GRS, MAJA and Sen2Cor corrections and mean reflectance measured by MODIS.

The reflectances of the Sentinel-2 products assessed from the three processings were also compared with those measured by MODIS sensor, products MOD09Q1 and MYD09Q1, for dates matching closely the Sentinel-2 satellite passages. The Sentinel-2 satellite images used in the study do not match exactly the MODIS image dates due to the presence of clouds and the closest dates to the 8-day composition were considered, with a maximum lag of two days and five days when used for comparison with S2 data and SSC samplings.

In this case, the study areas were reduced to the sample regions named U1 (Upstream) and D1 (Downstream) and IGP (Igarapé) for mean reflectance sampling (Figure 1).

The location of the U1 and D1 sampling regions was chosen due to the larger fraction of pure water pixels, the location of the HPP dam and the proximity of the SO HYBAM sediment monitoring station.

When comparing the reflectances obtained in the U1, D1 and IGP masks, similar to what occurred with the L1 mask, the GRS image pixel count on 01/19/2018 differs from the other two corrections, with 13,908 pixels in the U1 sample region, 1,914 pixels in the D1 sample region and 3,111 pixels in the IGP sample region, while Sen2Cor and MAJA products total $13,978,1,909$ and 3,114 pixels each, respectively, for the same regions. This difference in sampled pixel numbers does not exceed $0.5 \%$ of the total number of valid pixels.

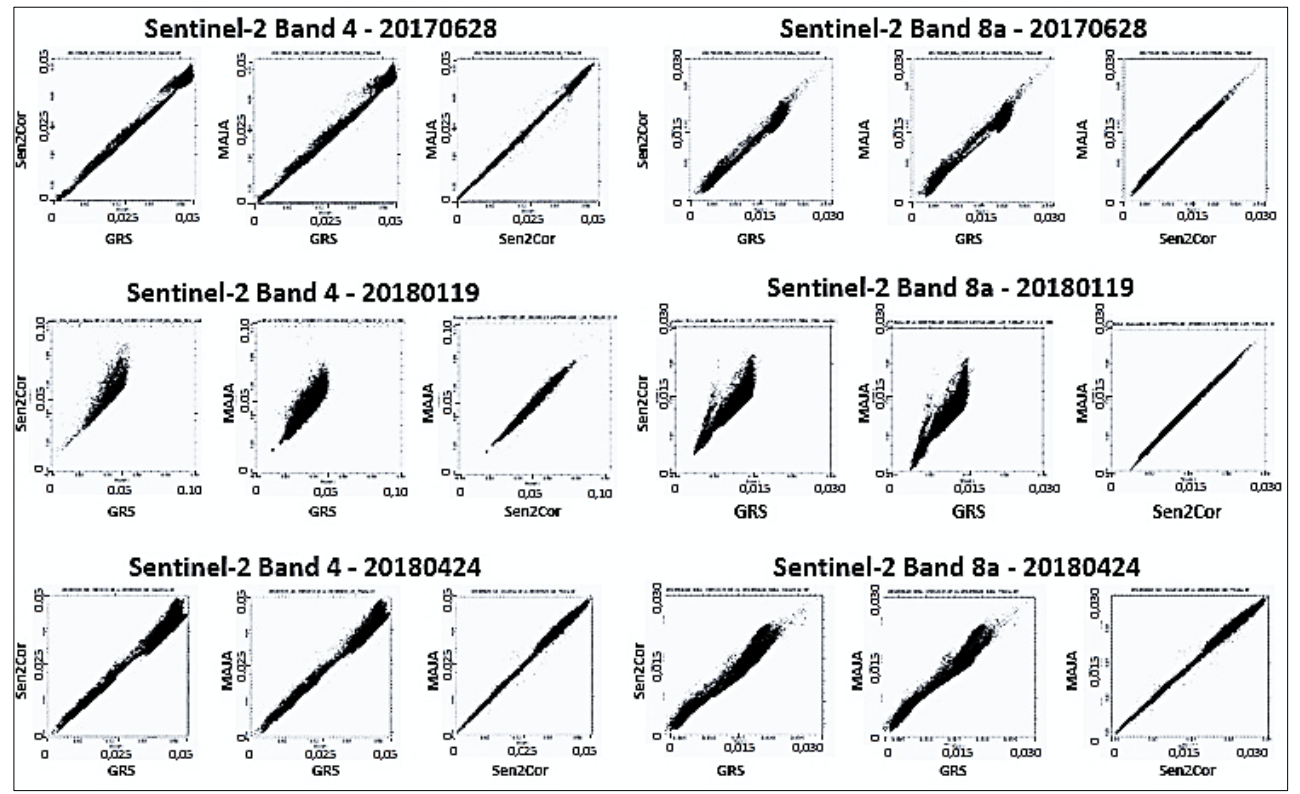

Figure 3 - Scattering diagrams of bands 4 and $8 \mathrm{a} \mathrm{S-2} \mathrm{reflectances} \mathrm{and} \mathrm{for} \mathrm{the} \mathrm{three} \mathrm{post} \mathrm{processing} \mathrm{considered}$ 
The MODIS sensor bands used for the following comparisons were the RED and IR bands, centered at $645 \mathrm{~nm}$ and $858.5 \mathrm{~nm}$, respectively, in the compositions dated $06 / 26 / 2017$ and $04 / 23 / 2018$. It is important to note that in addition to the dates and spatial resolutions, the widths and center of bands between the highlighted sensors are also different. The average reflectance values for each of them in each of the regions defined by the U1, D1 and IGP masks, as well as the reflectance values of Sentinel2 images according to the different atmospheric corrections are shown in Table 2.

Regarding the differences in the reflectances of the 4 Sentinel-2/MSI bands produced by each atmospheric correction in relation to the RED band of the MODIS sensor, the GRS product show an average difference of $32.8 \%$. However, the difference values only exceed $20 \%$ in January 2019 for U1 (23.3\%) and D1 (60.8\%) and in April 2018 for IGP (73.3\%), lower than what was measured by MODIS. For MAJA and Sen2Cor products only in April 2018, in the IGP region, the difference was over $20 \%$, which was respectively $31.3 \%$ and $28.9 \%$ underestimated in relation to MODIS measurements.

Nevertheless, when looking at the average reflectance results, it appears that the GRS correction products result in lower values than the others, in most cases, owing most probably to the the sunglint removal correction considered by GRS.

Observing the differences between band $8 \mathrm{a}$ Sentinel-2 / MSI to the IR band of the MODIS sensor, there is a strong inconsistency in relation to the band 4 results since only in U1 region there were differences below 20\% for June 2017 and April 2018, and in January 2019 only the Sen2Cor product showed values similar to those of MODIS products. Abrupt differences were found in the near-infrared region between the reflectances of these products, sometimes reaching differences between 500 and $1,000 \%$. In most cases the reflectance values of Sentinel-2 products with the applied atmospheric corrections were lower when compared to the MODIS reflectance values.

When Sentinel-2 band 4 results are analysed, over the U1 and D1 regions, the MAJA reflectance values are the highest among the three Sentinel-2 / MSI products in June, while in April the highest Sentinel-2 reflectance values come from Sen2Cor. Over the IGP region, the Sen2Cor processing show the largest reflectances for both dates. The reflectance values from GRS processing are always the smallest.

\subsection{Estimates of Suspended Sediment Concentration (SSC)}

SSC (Table 3) in each of the sample regions was estimated from Sentinel-2 / MSI reflectance data for each of the atmospheric correction processings by applying Eq. (2) and (3), which were developed specifically for each sensor's data.

Subsequently, the SSC estimates were compared to each other and each compared to the suspended sediment concentrations obtained by the HYBAM in situ monitoring station, located $5 \mathrm{~km}$ downstream of the Santo Antônio HPP, on the dates closest to the Sentinel-2 image acquisition and with best quality image for the 8-day MODIS composition.

The HYBAM monitoring network collect water samples since 2003 with measurements every 10 days. Figures $4 \mathrm{a}, 4 \mathrm{~b}$ and $4 \mathrm{c}$ show the variation of sediment concentrations measured at station 15400000 .

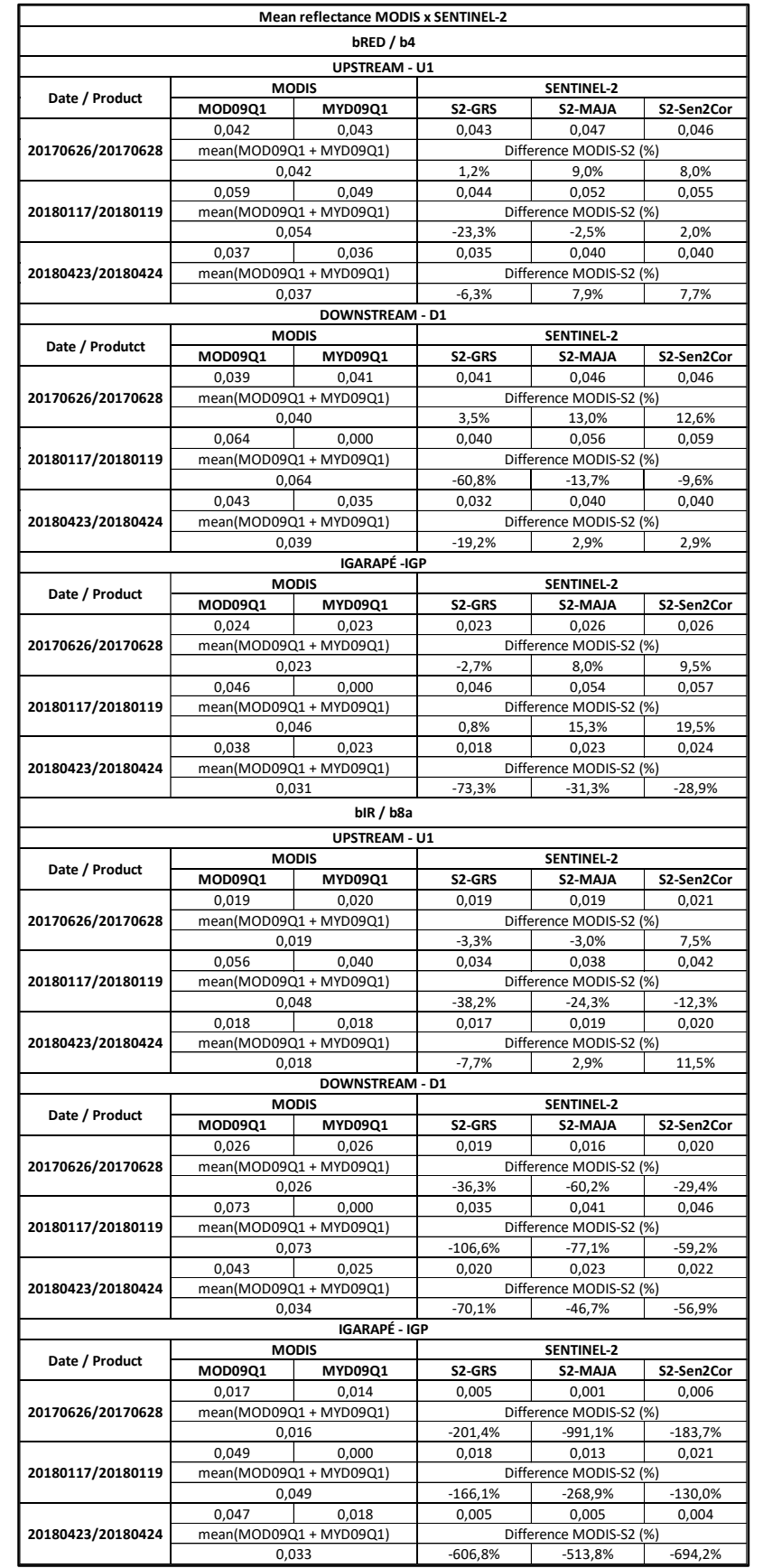

Table 2. Average reflectances values in M1, J1 and IGP regions

\begin{tabular}{|c|c|c|c|c|c|c|c|}
\hline \multicolumn{8}{|c|}{ SSC estimated by satelite data x HYBAM monitoring station $(\mathrm{mg} / \mathrm{L}$ ) } \\
\hline \multicolumn{8}{|c|}{ Upstream-U1 } \\
\hline Date & S2-GRS & S2-MAAA & S2-Sen2Cor & Data & MODIS & Data & HYBAM \\
\hline 20170628 & 191,1 & 163,7 & 206,6 & 20170626 & 104,1 & 20170623 & 378,0 \\
\hline 20180119 & 598,1 & 513,6 & 572,4 & 20180117 & 714,8 & 20180120 & 723,2 \\
\hline 20180424 & 228,5 & 211,8 & 254,7 & 20180423 & 124,2 & 20180420 & 145,4 \\
\hline \multicolumn{8}{|c|}{ Downstream - D1 } \\
\hline Date & S2-GRS & S2-MAAA & 52-Sen2Cor & Data & MODIS & Data & HYBAM \\
\hline 20170628 & 206,5 & 123,3 & 188,4 & 20170626 & 282,7 & 20170623 & 378,0 \\
\hline 20180119 & 745,4 & 512,2 & 586,8 & 20180117 & 1488,9 & 20180120 & 723,2 \\
\hline 20180424 & 365,5 & 326,8 & 286,5 & 20180423 & 690,4 & 20180420 & 145,4 \\
\hline \multicolumn{8}{|c|}{ SSC mean U1 D1 } \\
\hline Date & 52-GRS & 52-MAIA & S2-Sen2Cor & Data & MODIS & Data & HYBAM \\
\hline 20170628 & 198,8 & 143,5 & 197,5 & 20170626 & 193,4 & 20170623 & 378,0 \\
\hline 20180119 & 671,8 & 512,9 & 579,6 & 20180117 & 1101,8 & 20180120 & 723,2 \\
\hline 20180424 & 297,0 & 269,3 & 270,6 & 20180423 & 407,3 & 20180420 & 145,4 \\
\hline
\end{tabular}

Table 3 - SSC estimated by satellite data X HYBAM monitoring estation $(\mathrm{mg} / \mathrm{L})$ 

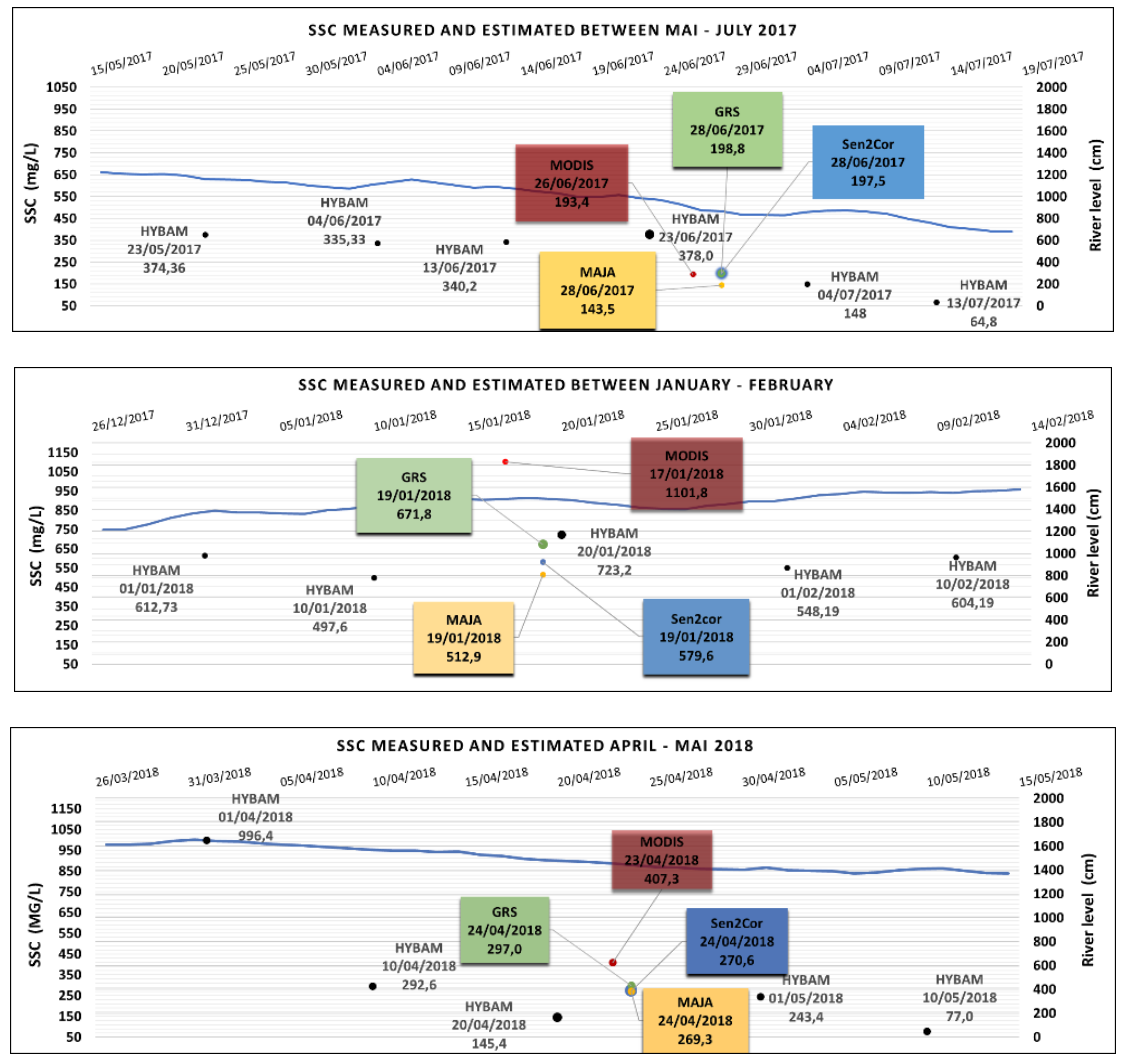

Figure $4 \mathrm{a}, 4 \mathrm{~b}$ e $4 \mathrm{c}-\mathrm{SSC}$ measured and estimated in tree distinct hydrological periods

The SSC maps (Figure 5) were produced from a clipping of the cloud mask from the MAJA product, however, disregarding the presence of cirrus clouds, thus covering the largest possible area according to the L1 region.

The SSC estimates with the results closest to those measured by the local station, according to the Sentinel-2/ MSI data, considering the average U1 D1, occurred when using the GRS atmospheric correction, with a $27.3 \%$ average difference from the HYBAM measurement, except for April, when all estimated satellite values exceeded an average of $92 \%$ those measured by the monitoring network. Consideration should be given to the time lag between the in situ measurement and the passing of the Sentinel-2 satellite, as well as the variation in the Madeira River water regime based on the time of the year, especially between March and April during the flood peak (Villar, 2013) during the SSC shows rapid variations.

A comparison of the results for each satellite product to each other shows that the best results occurred for U1 station in January 2018, when the difference was $19.5 \%$. The GRS product result was underestimated in relation to the estimate made with MODIS products, and the U1 D1 average in June 2017, which showed only a $2.1 \%$ difference (Table 4 ).

Differences in satellite derived SSC estimates were also compared between the results of the regions U1, D1 and the U1 D1 average (Table 5). In April 2018, all estimates presented the largest differences in relation to the regions. Regarding estimates made with MODIS products, the largest differences also occurred for the same date. Also, the estimates made with GRS atmospheric correction were always higher in the sample region D1 in relation to $\mathrm{U} 1$ and the $\mathrm{U} 1 \mathrm{D} 1$ average.

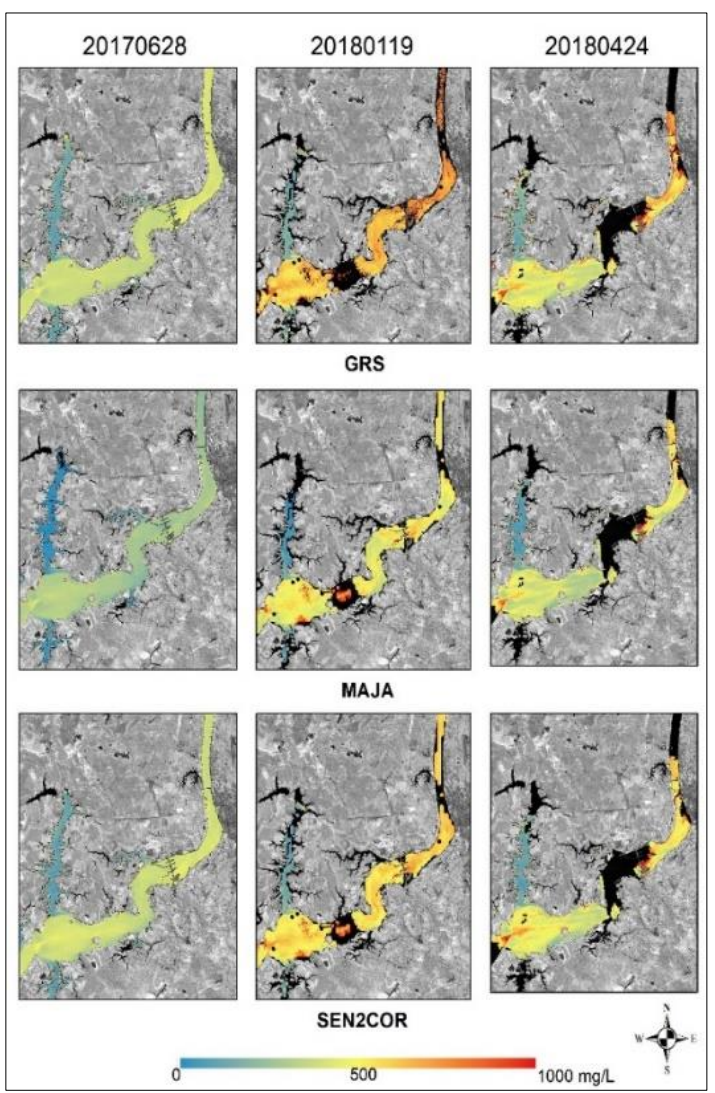

Figure 5 - Suspended sediment concentration estimates at the L1 sampling region (HPP Santo Antonio Reservoir) based on Sentinel-2 data using the three different atmospheric correction products. 


\begin{tabular}{|c|c|c|c|c|c|c|c|c|c|}
\hline \multirow[b]{2}{*}{ Date } & \multicolumn{3}{|c|}{ Upstream-U1 } & \multicolumn{3}{|c|}{ Downstream-D1 } & \multicolumn{3}{|c|}{ mean U1-D1 } \\
\hline & \multicolumn{9}{|c|}{ HYBAM monitoring station $x$ MODIS } \\
\hline $20170623 / 20170626$ & \multicolumn{3}{|c|}{$72,5 \%$} & \multicolumn{3}{|c|}{$25,2 \%$} & \multicolumn{3}{|c|}{$48,8 \%$} \\
\hline 20180120/20180117 & \multirow{2}{*}{\multicolumn{3}{|c|}{$\begin{array}{l}1,2 \% \\
14,6 \%\end{array}$}} & \multirow{2}{*}{\multicolumn{3}{|c|}{$-105,9 \%$}} & \multicolumn{3}{|c|}{$-52,4 \%$} \\
\hline $20180420 / 20180423$ & & & & & & $-374,8 \%$ & \multicolumn{3}{|c|}{$-180,1 \%$} \\
\hline \multirow{3}{*}{ Date } & \multicolumn{9}{|c|}{ HYBAM monitoring station X Sentinel-2 by atmospheric correction } \\
\hline & \multicolumn{3}{|c|}{ Upstream-U1 } & \multicolumn{3}{|c|}{ Downstream - D1 } & \multicolumn{3}{|c|}{ mean U1-D1 } \\
\hline & S2-GRS & 52-MAIA & S2-Sen2Cor & 52-GRS & 52-MAA & 52-Sen2Cor & S2-GRS & S2-MAA & 52-Sen2Cor \\
\hline $20170623 / 20170628$ & $49,4 \%$ & $56,7 \%$ & $45,4 \%$ & $45,4 \%$ & $67,4 \%$ & $50,2 \%$ & $47,4 \%$ & $62,0 \%$ & $47,8 \%$ \\
\hline 20180120/20180119 & $17,3 \%$ & $29,0 \%$ & $20,8 \%$ & $-3,1 \%$ & $29,2 \%$ & $18,9 \%$ & $7,1 \%$ & $29,1 \%$ & $19,9 \%$ \\
\hline $20180420 / 20180424$ & $-57,2 \%$ & $-45,6 \%$ & $-75,2 \%$ & $-151,4 \%$ & $-124,8 \%$ & $-97,1 \%$ & $-104,3 \%$ & $-85,2 \%$ & $-86,1 \%$ \\
\hline \multirow{3}{*}{ Date } & \multicolumn{9}{|c|}{ Sentinel- 2 by atmospheric correction $x$ MODIS } \\
\hline & \multicolumn{3}{|c|}{ Upstream-U1 } & \multicolumn{3}{|c|}{ Downstream - D1 } & \multicolumn{3}{|c|}{ mean U1-D1 } \\
\hline & S2-GRS & 52-MAIA & S2-Sen2Cor & S2-GRS & 52-MAA & 52-Sen2Cor & S2-GRS & 52-MAIA & 52-Sen2Cor \\
\hline $20170626 / 20170628$ & $45,5 \%$ & $36,4 \%$ & $49,6 \%$ & $-36,9 \%$ & $-129,3 \%$ & $-50,0 \%$ & $2,7 \%$ & $-34,8 \%$ & $2,1 \%$ \\
\hline $20180117 / 20180119$ & $-19,5 \%$ & $-39,2 \%$ & $-24,9 \%$ & $-99,7 \%$ & $-190,7 \%$ & $-153,7 \%$ & $-64,0 \%$ & $-114,8 \%$ & $-90,1 \%$ \\
\hline $20180423 / 20180424$ & $45,6 \%$ & $41,3 \%$ & $51,2 \%$ & $-88,9 \%$ & $-111,3 \%$ & $-141,0 \%$ & $-37,1 \%$ & $-51,3 \%$ & $-50,5 \%$ \\
\hline
\end{tabular}

Table 4 - Difference between estimated suspended sediment concentrations and HYBAM measurements

\begin{tabular}{|c|c|c|c|c|c|c|}
\hline \multirow{2}{*}{ Date } & \multicolumn{3}{|c|}{ Difference Sentinel-2/MSI } & \multirow[b]{2}{*}{ Date } & \multirow{2}{*}{ ifference MODI } & \multirow{2}{*}{$\begin{array}{l}\text { Reference region to calculate } \\
\text { the difference }\end{array}$} \\
\hline & S2-GRS & S2-MAJA & S2-Sen2Cor & & & \\
\hline 20170628 & $7,5 \%$ & $-32,8 \%$ & $-9,6 \%$ & 20170626 & $63,18 \%$ & \multirow{3}{*}{ Dif. U1xD1 (Ref. J1) } \\
\hline 20180119 & $19,8 \%$ & $-0,3 \%$ & $2,4 \%$ & 20180117 & $51,99 \%$ & \\
\hline 20180424 & $37,5 \%$ & $35,2 \%$ & $11,1 \%$ & 20180423 & $82,01 \%$ & \\
\hline 20170628 & $-4,0 \%$ & $12,4 \%$ & $4,4 \%$ & 20170626 & $-85,81 \%$ & \multirow{3}{*}{ Dif. U1 x mean U1D1 (Ref. M1) } \\
\hline 20180119 & $-12,3 \%$ & $0,1 \%$ & $-1,3 \%$ & 20180117 & $-54,15 \%$ & \\
\hline 20180424 & $-30,0 \%$ & $-27,2 \%$ & $-6,2 \%$ & 20180423 & $-227,87 \%$ & \\
\hline 20170628 & $3,7 \%$ & $-16,4 \%$ & $-4,8 \%$ & 20170626 & $31,59 \%$ & \multirow{3}{*}{ Dif. D1 x mean U1D1 (Ref. J1) } \\
\hline 20180119 & $9,9 \%$ & $-0,1 \%$ & $1,2 \%$ & 20180117 & $26,00 \%$ & \\
\hline 20180424 & $18,7 \%$ & $17,6 \%$ & $5,6 \%$ & 20180423 & $41,00 \%$ & \\
\hline
\end{tabular}

Table 5 - Difference between estimated suspended sediment concentrations by sampled region and date

\section{CONCLUSIONS}

The region in which the study took place is located in an environment with substantial cloud cover during most of the year, specially between November and April, which interferes with the acquisition of images of the whole reservoir.

The sediment data of the HYBAM monitoring station presented the solid load dynamics in the period between January and April 2018, which alternated between high and low loads, with a higher concentration in relation to June 2017, allowing to see also which hydrological period most influences the reflectance signal, since, following the SSC increase in January 2018, all products presented the highest values.

The differences in SSC estimates between those made by Sentinel-2 / MSI and the GRS and MODIS correction averaged $34.6 \%$. Overall, the SSC estimates from the GRScorrected algorithm images were closer to that measured by the HYBAM station (27.3\%), indicating that this is the most efficient atmospheric correction product for estimating suspended sediment in the studied area, as it is also capable of removing the solar reflection signal from water leaving radiance.

Given the differences presented, it is critical to keep in mind that SSC measurements taken every 10 days by the HYBAM monitoring network are point wise estimates, while satellite data estimates refer to averages for broader regions, given the spatial resolutions of the images offered by the orbital sensors addressed in this study. However, given the time lapse between satellite measurements and passages, remote sensing estimates have been shown to be coherent as they follow the trends shown in the historical SSC $x$ Level hydrosedimentological time-series.

The MODIS reflectance values, as well as the reflectances of the Sentinel-2 / MSI products for each of the atmospheric corrections, on the analysed dates, were on average
$11.4 \%$ different between band 4 RED and $36.3 \%$ between band $8 \mathrm{a} / \mathrm{IR}$. The red region showed greater congruence.

The products of the MAJA and Sen2Cor corrections were the most similar to each other, with MAJA showing reflectance values on average $6 \%$ lower than those of Sen2Cor processing, and the GRS correction most distinct among the three analysed, with lower reflectance on average of $13,4 \%$ and $20.2 \%$ in relation to MAJA and Sen2Cor, respectively, confirming its ability for sunglint removal.

\section{ACKNOWLEDGEMENTS}

This study was financed in part by the Coordenação de Aperfeiçoamento de Pessoal de Nivel Superior - Brasil (CAPES) - Finance Code 001. We had the contribution of Henrique Bernini who supported us with data, equations and discussions developed in his doctoral dissertation. Thanks also to the National Observatory SO HYBAM and the Institut de Recherche pour le Développement (IRD) for making the sample data available, to the Federal University of Amazonas (UFAM) and to the Institute of Geosciences of the University of Brasilia (IG-Unb).

\section{REFERENCES}

Cavalli, M. et al. Mapping water and sediment connectivity. Science of the Total Environment. Volume 673, 10 July 2019, 763-767. doi.org/10.1016/j.scitotenv.2019.04.0712019.

Hagolle, O. et al. MAJA Algorithm Theoretical Basis Document. Zenodo. (Version 1.0), December 2017 http://doi.org/10.5281/zenodo.1209633

Harmel, T. et al. Sunglint correction of the Multi-Spectral Instrument (MSI)-SENTINEL-2 imagery over inland and sea waters from SWIR bands. Remote Sensing of Environment.
Volume
204, January
2018 ,
$308-321$ doi.org/10.1016/j.rse.2017.10.022.

Martinez, J. M. et. al, The optical properties of river and floodplain waters in the Amazon River Basin: Implications for satellite-based measurements of suspended particulate matter. Journal of Geophysical Research: Earth Surface 120(7). 2015. 10.1002/2014JF003404.

Müller-W, U. Sentinel-2 MSI-Level 2A Products Algorithm Theoretical Basis Document; ESA Report 2012, ref S2PADATBD-0001 Issue 2.0; Telespazio VEGA Deutschland GmbH, 2015.

Ogashawara, I. Terminology and classification of bio-optical algorithms. Remote Sensing Letters, 6:8, 613617, DOI: 10.1080/2150704X.2015.1066523.

Palmer, S. C.J, et al. Remote sensing of inland waters: Challenges, progress and future directions. Remote Sensing of Environment. Volume 157, February 2015. 1-8 2014 doi.org/10.1016/j.rse.2014.09.021.

Villar R. E. et al. A study of sediment transport in the Madeira River, Brazil, using MODIS remote-sensing images. Journal of South American Earth Sciences. Volume 44, July 2013. 45-54. doi.org/10.1016/j.jsames.2012.11.006. 